\title{
Elliptic Biquadratic Sections Using Second Generation Current Conveyors (CCIIs)
}

\author{
Luka Šesnić and Dražen Jurišić \\ Faculty of Electrical Engineering and Computing \\ University of Zagreb \\ Unska 3, HR-10000 Zagreb, Croatia \\ e-mail: \{luka.sesnic | drazen.jurisic\}@fer.hr
}

\author{
Budimir Lutovac \\ Faculty of Electrical Engineering \\ University of Montenegro \\ George Washington bb, ME-81000 Podgorica, Montenegro \\ e-mail: budo@ac.me
}

\begin{abstract}
In this paper we propose a new, programmable, easytunable, current-mode general-purpose Biquad (C-GP-B), using second-generation current conveyors (CCIIs), based on the wellknown direct form II, which, among other transfer functions, is also suitable to realize band-rejection (BR) or notch transfer function. It is convenient for elliptic transfer function realizations because it can realize finite transfer function zeroes. Since it has low sensitivity and grounded capacitors it is suitable for integrated circuit (IC) design. The new Biquad is then compared in sensitivity performance with other two CCII-based versions of biquadratic sections obtained by different approaches. Since all presented Biquads use CCIIs they operate in the current mode, and are therefore suitable to realize filter circuits operating at higher frequencies, consuming less power, and needing lower supply voltage. As an example, the design procedure, and the Monte Carlo sensitivity analysis using PSpice were performed for a fourth-order elliptic low-pass (LP) filter.
\end{abstract}

Keywords-current mode filters; general-purpose Biquad; second-generation current conveyor; elliptic filters; low sensitivity.

\section{INTRODUCTION}

In the current-mode approach (e.g. using current conveyors) high-frequency filter circuits are feasible because there is no limitation on the gain-bandwidth product (GBW product) as it is present in the voltage-mode approach using operational amplifiers. Besides, circuits with good performance in terms of speed, bandwidth and accuracy are provided. They consume less power and need lower voltage for operation. The second-generation current conveyor (CCII) is considered as the basic active circuit building block for realizing the currentmode filters [1][2].

In this paper we propose a new current-mode, generalpurpose Biquad (C-GP-B) based on the well-known direct realization II, which is easy tunable, easy designable, realized by CCIIs, (grounded) capacitors and resistors. The new Biquad can be programmed using MOS switches to choose a desired transfer function characteristic. In the examples we realize the elliptic transfer function, and therefore we will need the bandrejection (BR) or notch transfer function. Because of lowsensitivity and grounded capacitors the proposed Biquad is capable for integrated circuit (IC) realization. The considered circuit is compared with two other common filter circuits satisfying the same filter specifications, but designed using different approaches. The sensitivity comparison of all circuits is performed by Monte Carlo runs using the PSpice 16 [3].

\section{GenERAL-PURPOSE BIQUAd In CURRENT Mode}

Fig. 1 shows a block diagram of the Biquadratic section with the current transfer function given by:

$$
H_{i}(s)=\frac{I_{\text {out }}(s)}{I_{\text {in }}(s)}=k \cdot \frac{b_{2} s^{2}+b_{1} \cdot s+b_{0}}{s^{2}+a_{1} \cdot s+a_{0}} .
$$

This structure is known as "direct form II" and provides all: low-, high-, band-, and all-pass, as well as band-rejection (notch) transfer functions characteristics; hence the section is referred to as general-purpose Biquad (GP Biquad).

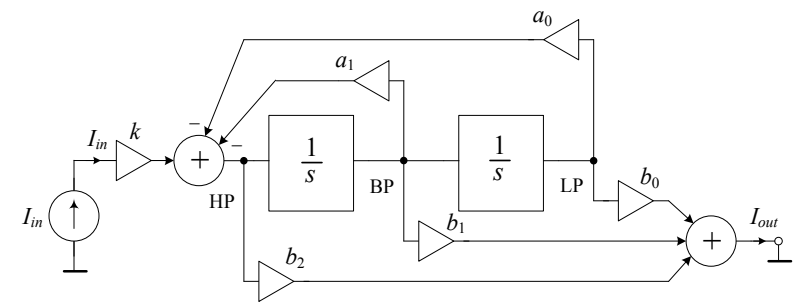

Figure 1. Block diagram of the GP Biquad in the current mode.

The diagram in Fig. 1 is applicable to both current and voltage modes. In the current mode the most simple is the realization of the summing functions. By simple application of the Kirchhoff's current law (KCL) the signals in the form of currents are summed in one node. This simplicity is not possible when summing signals carried by voltages in the voltage mode, because the summing circuit (consisting of resistors and additional active element, such as, e.g. operational amplifier) is required. On the contrary, signals at the outputs of integrators, if they were in the voltage mode, are simple to be conveyed from one node to other blocks of the diagram by simply connecting the wires to the outputs. In Fig. 1 the signals at the integrator outputs are in the current mode and it is rather complicated to convey current signals to different parts in the diagram. To propagate current signals, additional circuitry is needed that consist of resistors and additional active elements, such as, e.g. current conveyors (see Fig. 2). So in the sense of circuit complexity, what appears to be a simpler solution in the 
voltage mode is the more complicated in the current mode, and vice versa. The new GP Biquad in current-mode (C-GP-B) using all positive CCIIs and based on the diagram in Fig. 1 is shown in Fig. 2. The current transfer function of the circuit is given by (2). It is also convenient to choose: $R_{3}=R_{11}$, $R_{5}=R_{7}=R_{13}, R_{9}=R_{15}$, to split the currents at the $z$-outputs of CCIIs no. 1, 3 and 5 into the same amounts, entering into the subsequent circuits. While using $R_{4}=R_{3} / 2, R_{6}=R_{5} / 3$ unity gains of the integrators are obtained. Special cases of transfer functions (with conditions) for the filter in Fig. 2 are in Table I.
Coefficient-to-component (relative) sensitivities for the filter in Fig. 2 are calculated in order to investigate the influence of changes in element values on the filter amplitude characteristic [4]. Coefficients $a_{i}, b_{j}(i=0,1 ; j=0,1,2)$ and gain $k$ in (1) as functions of elements $R_{k}$ and $C_{l}(k=1, \ldots, 16 ; l=1,2)$ are obtained by comparing (1) and (2). Coefficient sensitivities are low; they are either -1 or $<1$. E.g., sensitivities $S_{R 1}^{a 0}=-1$, $S_{R 3}^{a 0}=\frac{R_{11}}{R_{3}+R_{11}}<1$ and $S_{R 5}^{a 0}=\frac{R_{7} R_{13}}{R_{5} R_{7}+R_{5} R_{13}+R_{7} R_{13}}<1$.

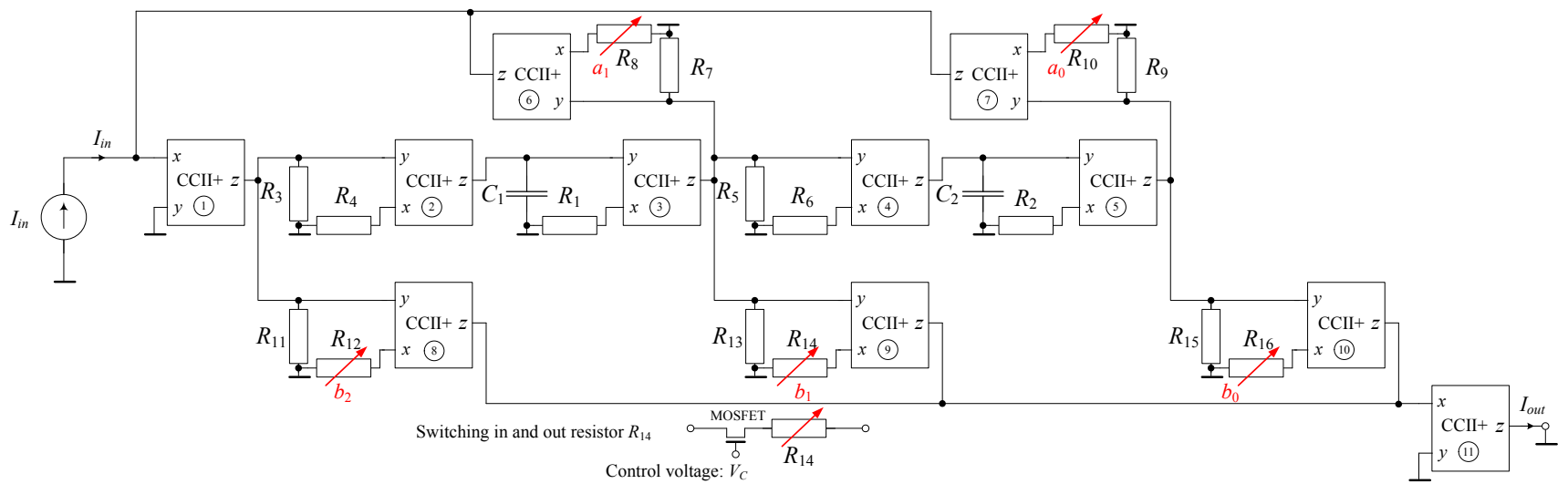

Figure 2. GP current-mode (programmable) Biquadratic section using all-positive CCIIs and grounded capacitors (C-GP-B).

$H_{i}(s)=\frac{I_{\text {out }}(s)}{I_{\text {in }}(s)}=\frac{s^{2} \frac{R_{3} R_{11}}{R_{12}\left(R_{3}+R_{11}\right)}+\frac{R_{3} R_{13} R_{11} R_{5} R_{7}}{R_{4} R_{14}\left(R_{3}+R_{11}\right)\left(R_{5} R_{7}+R_{5} R_{13}+R_{7} R_{13}\right) R_{1} C_{1}} s+\frac{R_{3} R_{5} R_{15} R_{9} R_{11} R_{7} R_{13}}{R_{4} R_{6} R_{16}\left(R_{9}+R_{15}\right)\left(R_{3}+R_{11}\right)\left(R_{5} R_{7}+R_{5} R_{13}+R_{7} R_{13}\right) R_{1} R_{2} C_{1} C_{2}}}{s^{2}+\frac{R_{3} R_{5} R_{9} R_{11} R_{5} R_{13} R_{13} R_{7} R_{13}}{R_{4} R_{8}\left(R_{3}+R_{11}\right)\left(R_{5} R_{7}+R_{5} R_{13}+R_{7} R_{13}\right) R_{1} C_{1}} s+\frac{R_{1}}{R_{4} R_{6} R_{10}\left(R_{9}+R_{15}\right)\left(R_{3}+R_{11}\right)\left(R_{5} R_{7}+R_{5} R_{13}+R_{7} R_{13}\right) R_{1} R_{2} C_{1} C_{2}}}$

TABLE I. TRANSFer Functions OF THE C-GP-BiQuAd IN Fig. 2.

\begin{tabular}{|c|c|c|}
\hline Realization & Transfer Function & Conditions \\
\hline Conjugate complex zeroes & {$\left[s^{2} \frac{R_{11}}{2 R_{12}}+s \frac{R_{13}}{3 R_{14} R_{1} C_{1}}+\frac{R_{15}}{2 R_{16} R_{1} R_{2} C_{1} C_{2}}\right] / D(s)$} & $\begin{array}{c}R_{3}=R_{11}=2 R_{4} ; R_{9}=R_{15} ; \\
R_{7}=R_{5}=R_{13}=3 R_{6}\end{array}$ \\
\hline High pass (HP) & $s^{2} \frac{R_{4}}{R_{12}} / D(s)$ & $R_{14} \rightarrow \infty, R_{16} \rightarrow \infty$ \\
\hline Band pass (BP) & $s \frac{R_{6}}{R_{14} R_{1} C_{1}} / D(s)$ & $R_{12} \rightarrow \infty, R_{16} \rightarrow \infty$ \\
\hline Low pass (LP) & $\frac{R_{15}}{2 R_{16} R_{1} R_{2} C_{1} C_{2}} / D(s)$ & $R_{12} \rightarrow \infty, R_{14} \rightarrow \infty$ \\
\hline $\begin{array}{c}\text { High pass with zeroes at } \\
j \omega \text {-axis (HP-Notch) }\end{array}$ & {$\left[s^{2} \frac{R_{4}}{R_{12}}+\frac{R_{15}}{2 R_{16} R_{1} R_{2} C_{1} C_{2}}\right] / D(s)$} & $R_{14} \rightarrow \infty, \omega_{p}>\omega_{z}$ \\
\hline $\begin{array}{c}\text { Low pass with zeroes at } \\
j \omega \text {-axis (LP-Notch) }\end{array}$ & {$\left[s^{2} \frac{R_{4}}{R_{12}}+\frac{R_{15}}{2 R_{16} R_{1} R_{2} C_{1} C_{2}}\right] / D(s)$} & $R_{14} \rightarrow \infty, \omega_{p}<\omega_{z}$ \\
\hline All pass (AP) & {$\left[s^{2} \frac{R_{4}}{R_{12}}-s \frac{R_{6}}{R_{14} R_{1} C_{1}}+\frac{R_{15}}{2 R_{16} R_{1} R_{2} C_{1} C_{2}}\right] / D(s)$} & CCII- (for coefficient $\left.-b_{1}\right)$ \\
\hline \begin{tabular}{c} 
Denominator \\
\hline
\end{tabular} & $D(s)=s^{2}+s \frac{R_{7}}{3 R_{8} R_{1} C_{1}}+\frac{2 R_{10} R_{1} R_{2} C_{1} C_{2}}{2}$ & \\
\hline
\end{tabular}




\section{A. Elliptic filter design using C-GP-B Biquad}

Using C-GP-Biquadratic section in Fig. 2 we have to realize LP elliptic filter. Since elliptic filters have zeroes on the $j \omega$-axis (purely imaginary zeroes), coefficient $b_{1}$ in (1) has to be zero, which is accomplished by "switching out" (opencircuiting) resistor $R_{14}$. By appropriately switching in and out the corresponding resistors (e.g. using MOS switches) different kind of transfer functions can be obtained using C-GP-B. Since output $x$ of CCII is a voltage sources, resistors connected to that source can be switched out using MOS switches; the current through $x$ connector is then equal to zero, the conveyed current on the $z$ output is also zero, and the signal is turned off. Different kinds of transfer functions obtained by switching-in and -out appropriate resistors are shown in Table I. To design elliptic filter the following resistor values have to be calculated:

$$
\begin{gathered}
R_{8}=R_{7} /\left(3 a_{1} R_{1} C_{1}\right) ; R_{10}=R_{9} /\left(2 a_{0} R_{1} C_{1} R_{2} C_{2}\right) ; R_{12}=R_{11} /\left(2 b_{2}\right) ; \\
R_{14}=R_{13} /\left(3 b_{1} R_{1} C_{1}\right) ; R_{16}=R_{15} /\left(2 b_{0} R_{1} C_{1} R_{2} C_{2}\right) .
\end{gathered}
$$

Note from (3) that each coefficient in the transfer function (1) can be realized simply by calculating the corresponding resistor. Note, also, that the resistors calculated in (3) are the same resistors that can be used for tuning the filter coefficients as shown in Fig. 2 (orthogonal tuning accurate filter parameters is also possible using programmable capacitive and resistive arrays). Tuning procedure is the same as for all direct-form-II type filters, and tuning range depends on the technology used. It is also convenient to choose $R_{7}=R_{9}=R_{11}=R_{13}=R_{15}=1$ then (3) becomes simpler, and we obtain the current transfer function in the BR form, given by:

$$
\begin{aligned}
& H_{i}(s)=I_{\text {out }}(s) / I_{\text {in }}(s)= \\
& =\frac{\frac{1}{2 R_{12}} \cdot\left(s^{2}+\frac{R_{12}}{R_{16} R_{1} R_{2} C_{1} C_{2}}\right)}{s^{2}+s \frac{1}{3 R_{8} R_{1} C_{1}}+\frac{1}{2 R_{10} R_{1} R_{2} C_{1} C_{2}}}=\frac{k \cdot\left(s^{2}+\omega_{z}^{2}\right)}{s^{2}+s \frac{\omega_{p}}{q_{p}}+\omega_{p}^{2}},
\end{aligned}
$$

with the parameters given by:

$$
\begin{gathered}
\omega_{p}=\left(2 R_{10} R_{1} R_{2} C_{1} C_{2}\right)^{-1 / 2} ; \\
\omega_{z}=\left[R_{12} /\left(R_{16} R_{1} R_{2} C_{1} C_{2}\right)\right]^{1 / 2} ; \quad k=\frac{1}{R_{12}} ; q_{p}=\frac{3 R_{8}}{\sqrt{2 R_{10}}} \sqrt{\frac{R_{1} C_{1}}{R_{2} C_{2}}} .
\end{gathered}
$$

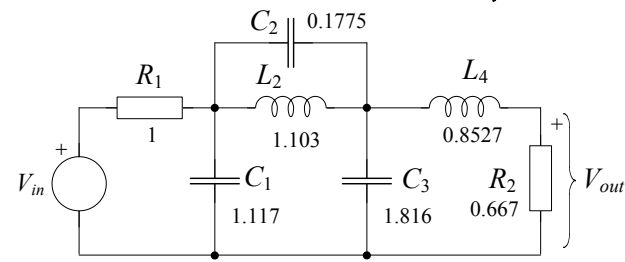

Figure 3. Passive (voltage-mode) ladder-RLC filter LP prototype.

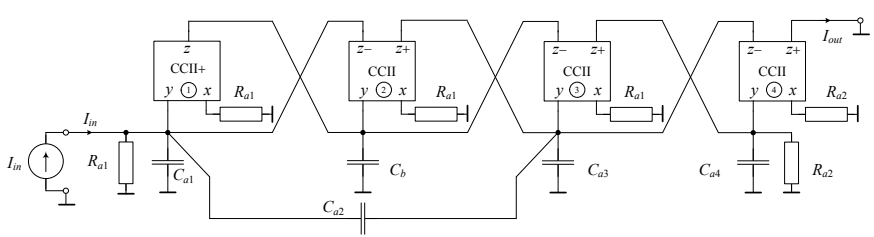

Figure 4. Active current-mode LP filter using MOCCII derived from LP single-ladder filter prototype in Fig. 3 (LIN-T-F).

\section{EXAMPLE}

We have to realize a LP filter that meets the following specifications: maximum pass-band attenuation $A_{\max }=0.2 \mathrm{~dB}$, minimum attenuation in the stop band $A_{\min }=40 \mathrm{~dB}$, pass-band cut-off frequency $f_{p}=10 \mathrm{kHz}$, and the stop-band cut-off $f_{s}=$ $20 \mathrm{kHz}$ (transition region width $\Omega_{s}=f_{s} / f_{p}=2.0$ ) [see Fig. 6]. Poles and zeroes of this filter can be obtained from filter tables [5], or can be calculated using MATLAB (command ellip). The name of the filter which satisfies specifications is CC 042036 $\mathrm{B}$; it is a type-B elliptical filter of the order $N=4$, with reflection factor $\rho=20 \%$ (corresponding to the maximum pass-band ripple $A_{\max }[\mathrm{dB}]=-10 \log \left(1-\rho^{2}\right)=0.17728767[\mathrm{~dB}]$ and angle $\Theta=36^{\circ}$, corresponding to the width of the transition region $\Omega_{s}=1.815103$, and has the minimum attenuation in the stop band $A_{\min }=40.53 \mathrm{~dB}$ [5]. It is evident that this filter meets the given specifications. The corresponding passive ladder-RLC filter is shown in Fig. 3 with the normalized elements obtained from [5], and with the normalized transfer function given by:

$$
H_{B}(s)=\frac{0.41710 s^{2}+0.08166}{s^{4}+1.56427 s^{3}+2.25045 s^{2}+1.63172 s+0.69531} .
$$

It is well-known that ladder-RLC filter in Fig. 3 has low sensitivity to passive components variations according to

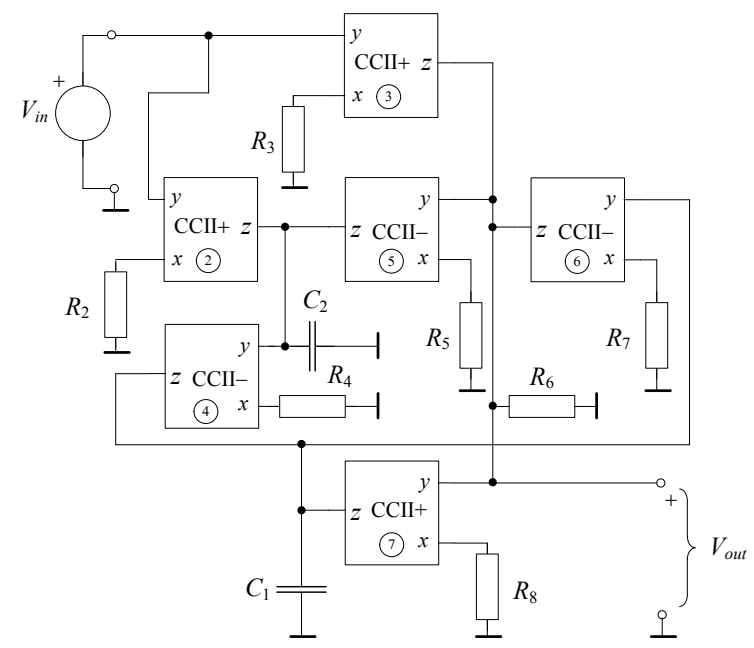

Figure 5. BR biquadratic section using CCII (Biquad in [9]).

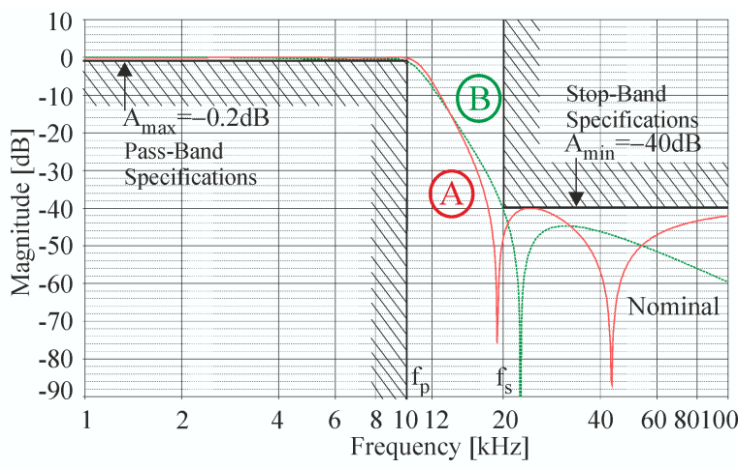

Figure 6. Amplitude characteristics of the current transfer function $H_{i}(s)$ of fourth-order LP filter examples. 

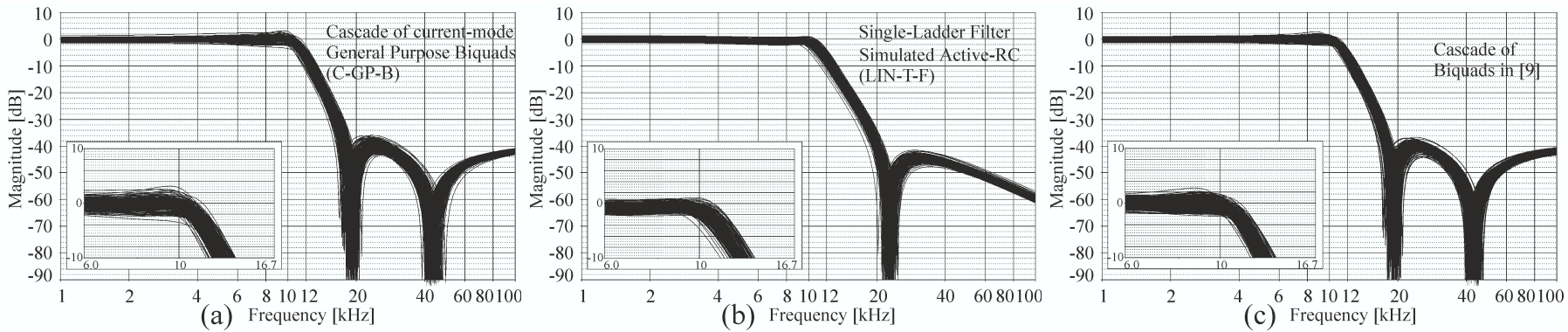

Figure 7. Monte Carlo runs of fourth-order elliptic LP filter realizations: (a) Filter obtained by cascading two C-GP-B Biquads (Fig. 2). (b) Active-RC simulated single-ladder filter by linear transformation (LIN-T-F) (Fig. 4). (c) Filter obtained by cascading two Biquads in [9] (Fig. 5).

Orchard's theorem [6] and that property is inherited to its active simulation, which is shown in Fig 4. Fig. 4 shows the current-mode active filter (using multiple-output CCIIs), derived using linear transformation (LIN-T-F) in [7][8], starting from the LP ladder RLC prototype circuit in Fig. 3.

For the even-order elliptical filter, there exist also filter type A (with two zeroes, which cannot be realized unless we have allowed transformers; allowing negative inductances). The filter's normalized transfer function of type A (generic) is obtained using MATLAB's function ellip and is given by:

$$
H_{A}(s)=\frac{0.01 \cdot\left(s^{2}+18.7940\right)\left(s^{2}+3.6676\right)}{\left(s^{2}+1.1520 s+0.5996\right)\left(s^{2}+0.3570 s+1.1765\right)} .
$$

Even-order elliptic filter of type A has two finite zeroes, while that of type B has one. Both transfer functions (6) and (7) are de-normalized to the cut-off $10 \mathrm{kHz}$ and theirs amplitude characteristics $\alpha(\omega)=20 \log |H(j \omega)|[\mathrm{dB}]$ are shown in Fig. 6 . We realized two types-A filters using circuits in Figs. 2 and 5. Finally, two filters in Figs. 4 and 5 using different topologies are compared with the proposed new C-GP-B filter in Fig. 2.

\section{A. Sensitivity Analysis}

We compare the sensitivity of three circuits (using CCIIs) in this paper, realizing fourth-order LP elliptic filters. In what follows, we examine the sensitivity to tolerances of passive components using the OrCAD PSpice 16 program [3] with Monte Carlo (MC) runs. We assume a zero-mean uniform distribution, and a 5\% standard deviation for all components. The sensitivity of the fourth-order LP filter using C-GP-B blocks (Fig. 2) is shown in Fig. 7(a), that of LIN-T-F (Fig. 4) in Fig. 7(b), and that using Biquads in [9] (Fig. 5) is shown in Fig. 7(c). In insets are the magnified results of the $\mathrm{MC}$ analysis around the cut-off frequency (near the cut-off frequency is always the highest sensitivity). As expected, the lowest sensitivity has LIN-T-F section. The highest (worst) has the cascade of two C-GP-B Biquads and the middle is the cascade of current Biquadratic sections (as in [9]), although we see that all three versions have very similar, low sensitivities. The sensitivity of the filter is determined by electrical filter topology and number of passive components, while the number of active elements does not affect sensitivity. The new currentmode C-GP-B section, though having large number of passive components, has low sensitivity due to the low-sensitivity of the transfer function coefficients to elements (see Section II).

\section{CONCLUSIONS}

In this paper we present a new current-mode generalpurpose Biquadratic section (C-GP-B) using CCIIs, based on common direct form II realization. We have compared its sensitivities to other two LP fourth-order current-mode filter circuits, all of them realizing elliptic transfer functions satisfying the same specifications. We have concluded that all three filters have equally low sensitivity to passive elements. Therefore, newly proposed filter section is very practical for building high-quality filters in integrated circuits (IC) form, because of low sensitivity and grounded capacitors. It has all the advantages of the current-mode approach: high frequency, low voltage, low-power operation, etc. Besides, it is easy to design, easy to tune, and is programmable to realize the desired transfer function using MOS switches.

\section{ACKNOWLEDGMENT}

This work has been financially supported by the bilateral project "Integrated Systems for Analog and Mixed Signal Processing-Active-RC filters for ADSL Communication Systems" between the Ministry of Science, Education and Sports of the Republic of Croatia and the Ministry of Science of the Republic of Montenegro.

\section{REFERENCES}

[1] C. Toumazou, F. J. Lidgey and D. G. Haig, Analog IC Design: The Current Mode Approach, London, U.K.: Peter Peregrinus, 1990.

[2] G. Ferri and N. C. Guerrini, Low Voltage, Low Power CMOS Current Conveyors, Boston, U.S.: Kluwer Academic Publishers, 2003.

[3] PSPICE Users Guide ver. 16.5, Cadence Design Systems, Inc. (Cadence), San Jose CA-USA, May 2011.

[4] R. Schaumann, M. S. Ghausi and K. R. Laker, Design of Analog Filters, Passive, Active RC, and SC, New Jersey: Prentice Hall, 1990.

[5] A. I. Zverev, "Handbook of Filter Synthesis", New York: Wiley, 1967.

[6] H. J. Orchard, Inductorless filters, Electronics Letters 1966, vol. 2, no. 6, pp. 224-225.

[7] L. A. Said, A. H. Madian, M. H. Ismail and A. M. Soliman, "Active realization of doubly terminated LC ladder filters using current feedback operational amplifier (CFOA) via linear transformation", Int. J. of Electronics and Communications 2011, vol. 65, no. 9, pp. 753-762.

[8] Y. S. Hwang, P. T. Hung, W. Chen and S. I. Liu, "Systematic Generation of Current-Mode Linear Transformation Filters Based on Multiple Output CCIIs", Analog Integrated Circuits and Signal Processing 2002, vol. 32, no. 2, pp. 123-134.

[9] L. Nedelea, M. Neag, M. Topa and L. Festila, "A new CCII-Based Universal Biquad", 2006 IEEE International Conference on Automation, Quality and Testing, Robotics, AQTR, (Cluj-Napoca, Romania), 25-28 May 2006, pp. 195-198. 